\title{
DISEÑO Y ELABORACIÓN DE UNA FERULA TIPO TENODESIS FABRICADA EN TERMOPLASTICO DE ALTA RESISTENCIA POLICLORURO DE VINILO P.V.C. PARA LA PROMOCION DE AGARRE DE PINZA TRIPODE EN PERSONAS CON SECUELAS DE TRAUMA RAQUIMEDULAR
}

\author{
DESIGN AND DEVELOPMENT OF A TENODESIS-TYPE SPLINT MADE IN HIGH RESISTANCE \\ THERMOPLASTIC POLYVINYL CHLORIDE (PVC) TO PROMOTE TRIPOD CLAMP GRIP IN PEOPLE \\ WITH SPINAL CORD TRAUMA AFTERMATH \\ Jorge Enrique Mayor B. ${ }^{1}$
}

\section{Resumen}

El presente documento es una propuesta teórica, que tiene como finalidad presentar un protocolo de elaboración de una férula dinámica tipo Tenodesis a partir de un material termoplástico de alta temperatura, de fácil consecución en el mercado llamado Policloruro de vinilo; Este tipo de férula se clasifica dentro de las denominadas férulas dinámicas y su objetivo final es la promoción de agarre de pinza trípode en personas que por lesiones a nivel de C5-C6, presentan ausencia de movilidad en los dedos de la mano pero conservan movimientos de muñeca. Cuando el usuario extiende la muñeca, la férula activa una serie de mecanismos que producen flexión pasiva de articulaciones metacarpofalangicas de los dedos II y III y simultáneamente hay una abducción y una relativa oposición del pulgar; estos movimientos formarán una pinza que proveerá al usuario la posibilidad de realizar actividades básicas con sus manos.

\section{Palabras clave}

Férula, tenodesis, policloruro de vinilo, trauma raquimedular, biomecánica, articulaciones metacarpofalangicas.

\footnotetext{
${ }^{1}$ Egresado Terapia Ocupacional Universidad del Valle. Colombia. Diseñador de dispositivos apoyo rehabilitación física: Férula tipo tenodesis fabricada en termoplástico de alta resistencia policloruro de vinilo p.v.c. para la promoción de agarre de pinza trípode en personas con secuelas de trauma raquimedular.

Férula/prótesis patrón tenodesis fabricada en termoplástico de alta resistencia policloruro de vinilo p.v.c. para la promoción de agarre de pinza trípode en personas con amputación de dedo i, dedos i y ii o dedos ii y iii Santiago de Cali. mayor310@hotmail.com
} 


\begin{abstract}
This document is a methodological contribution, which is to present a protocol for developing a Tenodesis type dynamic splint from a high temperature thermoplastic material, easy to achieve on the market called polyvinyl chloride, this type of splint is classified within the so-called dynamic splints and its purpose is to promote tripod pincer grip in people with injuries at $\mathrm{C5}-\mathrm{C6}$, present lack of mobility in the fingers but retain wrist movements. When the user extends the wrist, splint activates a series of mechanisms that produce passive flexion of metacarpophalangeal joints of fingers II and III and simultaneously there is a relative abduction and thumb opposition, these movements form a clamp that will provide the user the ability to perform basic activities with their hands.
\end{abstract}

\title{
Key words
}

Splint tenodesis, polyvinyl chloride, spinal cord injury, biomechanics, metacarpophalangeal joints. 


\section{INTRODUCCIÓN}

El presente documento, es un aporte tecnológico que tiene como finalidad presentar el protocolo de elaboración de una férula dinámica tipo Tenodesis elaborada a partir de un material termoplástico de alta temperatura de fácil consecución en nuestro medio, denominado Policloruro de Vinilo (PVC.); con él , se pretende re-plantear la elaboración de un dispositivo terapéutico (poco utilizado en nuestro medio debido a la alta dificultad para elaborarlo) que promueve el agarre de pinza trípode en personas que presentan secuelas de trauma raquimedular, esta propuesta a su vez representa bajo costo con respecto a otros materiales utilizados para la realización de férulas o dispositivos terapéuticos; valga aclarar que el dispositivo elaborado en este termoplástico proporciona un alto nivel de funcionalidad en lo relativo a su manejo y adaptabilidad tanto en el quehacer profesional del terapeuta ocupacional como para el usuario,

El dispositivo adaptado descrito en este artículo, busca compensar la gran dificultad que presenta quien ha padecido lesiones a nivel de C5-C6 para realizar sus Actividades de la Vida Diaria, llegando a ser dependientes hasta un cien por ciento de su cuidador, debido en parte, a no lograr realizar agarres de manera activa, bajo esta premisa lo que se busca es proporcionar un dispositivo liviano, estético, económico y de fácil mantenimiento, que permita aprovechar al máximo las capacidades que la persona conserva, mediante la utilización de nuevas técnicas y adaptaciones en la búsqueda de movimientos activos de la mano que permitan que logren en su proceso de rehabilitación, es decir, ejecutar actividades cotidianas tales como comer, cepillar sus dientes, peinarse $\mathrm{y} / \mathrm{o}$ afeitarse entre otras, que requieren un agarre estable, firme y funcional

\section{MARCO CONCEPTUAL}

\section{Efecto Tenodesis}

Las personas que han sufrido una lesión completa de la médula espinal en C5 C6 suelen presentar un movimiento «falso» de tenodesis como resultado del encogimiento natural de los tendones flexores (TURNER, 2002). Esto puede aumentar la dependencia física del sujeto y dificultarle el uso de ayudas técnicas. La extensión de la muñeca provoca la flexión natural de los dedos y la aducción y flexión del pulgar. La flexión de la muñeca hace que los dedos se extiendan en todas las articulaciones y provoca la abducción y extensión del pulgar; Si bien la Tenodesis ocurre de forma natural a veces es necesario facilitarla para asegurarse que los tendones realicen contracción con eficacia, específicamente los 
tendones flexores del pulgar. Las férulas combinadas con el movimiento pasivo, son una forma de lograr este objetivo.

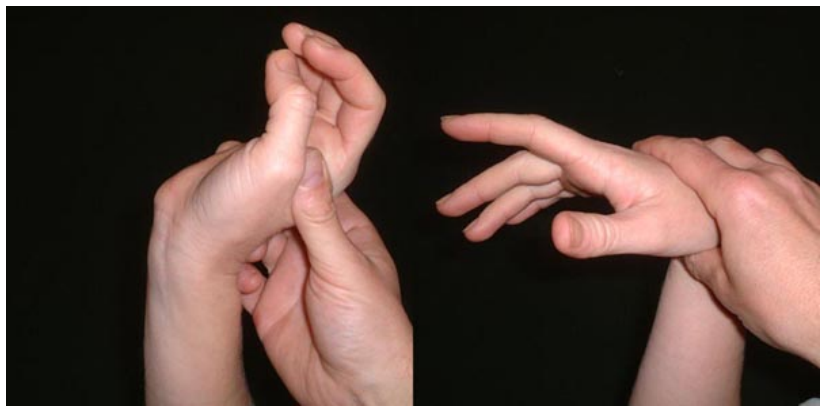

Figura 1. patrón pasivo de Tenodesis

Ahora bien, se hace necesario aclarar que una Férula Tipo Tenodesis es un dispositivo que facilita el patrón de agarre a mano llena, para las personas que presentan secuelas de cuadriplejia o lesiones a nivel de C5-C6, y que no logran realizar un movimiento voluntario de los dedos pero conservan la capacidad de flexionar y extender su muñeca. En cuanto al dispositivo, este abarca el antebrazo, la mano y los dedos I a III.

Este tipo de férula promueve una ligera extensión de la muñeca o en su defecto, evitar la flexión de la muñeca, por lo tanto, ayuda a recuperar la funcionalidad de aquellas articulaciones que se encuentran comprometidas a través un patrón de agarre por un efecto de Tenodesis.

La férula Tenodesis permite al usuario, alcanzar cierta independencia en actividades de higiene, de escritura, y de alimentación. Esta férula está indicada para personas con lesión de la médula espinal completa en $\mathrm{C} 6$ sin movimientos conservados de flexión o extensión de los dedos o en casos de tetraplejia completa (MALKUSH, 1998).

En relación a lo anterior, para la construcción de dispositivo objeto del presente proyecto se hizo necesario una revisión puntual de algunos marcos de referencia y principios de construcción, la cual presento a continuación a manera de breve resumen: 


\section{El Marco de Referencia Biomecánico}

Este marco se basa en cuatro suposiciones básicas (TROMBLY, 1990):

1. La creencia de que las actividades con objetivo pueden utilizarse para tratar la pérdida del arco de movimiento, la fuerza y la resistencia.

2. Admitir que, luego de recuperar el arco de movimiento, la fuerza y la resistencia, el usuario automáticamente recupera la función.

3. El principio de reposo y estrés. Primero el cuerpo reposa para recuperarse. Luego, las estructuras periféricas deben estar en estrés para recuperar el arco, la fuerza y resistencia. 4. El Marco de Referencia Biomecánico beneficia más a aquellos usuarios con un sistema nervioso central intacto. Los usuarios pueden tener limitación del arco, fuerza y resistencia, pero tener habilidad para desempeñar movimientos aislados y suaves.

El comportamiento del usuario puede determinarse al encontrar comportamientos indicadores de cambio en la evaluación de:

- La estabilidad estructural

- El bajo nivel de resistencia

- El control del edema

- El arco de movimiento pasivo

- La fuerza y el alto nivel de resistencia

\section{El Marco de Referencia de Rehabilitación}

Este marco enseña al usuario a compensar los déficit subyacentes que no pueden remediarse (HOPKINS, SMITH 2001). Contiene cinco suposiciones:

1. Establece que una persona puede recuperar la independencia a través de la compensación.

2. La motivación para la autonomía no puede separarse de los subsistemas volitivos y habituación. La motivación para la autonomía está influenciada por los valores de toda la vida, los roles futuros y un renovado sentido de propósito.

3. La motivación para la autonomía no puede separarse del contexto ambiental. Las demandas del ambiente de alta, la situación económica del usuario y los recursos emocionales de la familia son ejemplos de las influencias ambientales sobre la motivación para la autonomía. 
4. Un mínimo de destrezas cognitivas y emocionales son necesarias para que la autonomía sea posible.

5. El razonamiento clínico debe hacer un enfoque de arriba abajo. Los cinco pasos de esta revisión de arriba abajo son:

- $\quad$ Las demandas del ambiente.

- La capacidad funcional actual.

- $\quad$ Las demandas de tareas que el usuario no puede desempeñar.

- $\quad$ El tipo de método de rehabilitación.

- Las modalidades específicas.

\section{Modelo de Actividad Humana Tecnología de Asistencia (AHTA)}

Para la prescripción de tecnología de asistencia, la Terapeuta Ocupacional Susan Hussey, conjuntamente con S.M. Cook desarrollaron un modelo teórico, basado en la teoría de factor humano, denominado "Modelo de Actividad Humana Tecnología de Asistencia" AHTA (LASERNA, 2002). Dicho modelo, muestra la tecnología de asistencia como la relación de cuatro componentes, la habilidad de la persona, el dispositivo de Tecnología de Asistencia, la actividad y el contexto. Cada componente juega un papel único en todo el sistema; el modelo se inicia con la necesidad de la persona para llevar a cabo o desempeñar una actividad como cocinar, escribir o jugar, necesidades que definen la meta. Cada actividad se lleva a cabo en un contexto el cual incluye aspectos sociales y culturales así como ambientales y condiciones físicas (temperatura, ruido, luminosidad); la combinación de esta actividad con el contexto determina cuales son las habilidades humanas que se necesitan para lograr las metas propuestas.

El Modelo de Actividad Humana Tecnología de Asistencia (AHTA) se entiende como un sistema que interrelaciona la interfase o mecanismo que facilita la interacción persona tecnología y establece un límite entre estas dos; esta interacción tecnología/persona, es un fenómeno, de doble vía, esto es, puede partir de la persona hacia la tecnología o viceversa.

\section{Principios Biomecánicos}

Considerando que las férulas requieren la aplicación directa y la manipulación de fuerzas externas, es importante comprender los principios mecánicos básicos para aplicarlos en su diseño y elaboración. Esto es fundamental, porque garantiza que las férulas sean más cómodas, duraderas y efectivas, disminuyendo así las oportunidades de riesgo adicional secundarias a la aplicación de este tipo de dispositivos (ACOSTA, 2007). 
Las consideraciones básicas son las siguientes:

Incremento del área de aplicación de la fuerza para dispersar la presión.

La presión es igual a la cantidad de fuerza dividida por el área de aplicación de la fuerza.

$$
\text { PRESIÓN }=\frac{\text { FUERZA_TOTAL }}{\text { AREA_DE_APLICACIOON } N_{-} D E_{-} L A_{-} F U E R Z A}
$$

Las aplicaciones clínicas de este principio son:

1. La presión y la fricción pueden reducirse incrementando el área de superficie de la férula y asegurando su solución de continuidad sobre el miembro.

2. Las férulas o los componentes pequeños generalmente son problemáticos porque aplican fuerza a un área pequeña, creando necrosis por presión de los tejidos blandos periféricos.

\section{Incremento de ventaja mecánica para reducir la presión y el confort}

La ventaja mecánica es igual a la fuerza dividida por la resistencia. Es la aplicación de un sistema de fuerzas paralelas donde la férula funciona como una palanca de primer género, con la articulación como eje, la parte distal ferulada es la resistencia $(R)$ y la parte proximal es la fuerza $(F)$. Los diseños de férulas donde hay un cubrimiento óptimo del segmento, incrementan las ventajas mecánicas.

\section{Asegurar tres puntos de presión}

Las férulas de tres (3) puntos funcionan mediante una serie de fuerzas reciprocas con la fuerza media dirigida en oposición a las dos fuerzas finales. Estas tres fuerzas paralelas tanto en un sistema de palanca de primer género como en una férula, se encuentran en equilibrio.

\section{Posición de la muñeca}

Esta es una consideración importante en el diseño de una férula. El agarre es más favorable cuando la muñeca esta ligeramente extendida, sin embargo, hay que considerar que las personas de dominancia derecha cuando escriben posicionan su muñeca en una ligera extensión, mientras aquellas que son de dominancia izquierda posicionan su muñeca en ligera flexión. Lo anterior, indica que las consideraciones individuales en cuanto a la 
posición de la muñeca, pueden ser primordiales en la fabricación de férulas para esta articulación.

Vale aclarar, que en el caso de la Férula de Tipo Tenodesis, esta articulación debe permanecer libre, considerando que, el dinamismo central de esta férula se realiza a partir de los movimientos de muñeca que pueda realizar el usuario.

\section{Principios de Diseño}

Los principios de diseño proporcionan el armazón básico en que se debe basar la fabricación de cualquier tipo de férula para mano, considerando que los principios específicos, pueden ser alterados por las variables individuales de los usuarios y los requisitos funcionales (ACOSTA, 2007), lo cual influye en la última configuración de la férula. El desafío es crear una férula que no sólo cumpla con los objetivos funcionales, sino que además ser aceptable y tolerada por el usuario.

\section{Principios Generales de Diseño}

Son aquellos que acogen los conceptos más generales y que se convertirán en datos e información importante para el diseño y elaboración de la férula, en la búsqueda de resultados funcionales y prácticos dirigidos tanto al usuario como al terapeuta ocupacional.

\section{Considerar factores individuales en el usuario}

Factores individuales en el usuario como edad, motivación, medidas corporales, nivel de actividad, la situación económica, el tiempo de uso de la férula y el programa de ejercicios que deberá realizar.

\section{Presentar un diseño sencillo y de aspecto agradable}

Las férulas deben ser lo más sencillas posible, para permitir el funcionamiento óptimo del miembro afectado. $Y$, teniendo en cuenta que estos dispositivos son de apariencia extraña, deben realizarse lo más estéticas posible, cuidando los detalles y los acabados.

\section{Permitir la función óptima de la extremidad}

La extremidad superior, tiene una habilidad única para desplazarse libremente en un amplio rango de movimiento, lo cual permite el cumplimiento satisfactorio de una variedad de tareas diarias. Los segmentos del brazo y mano funcionan como una cadena cinética abierta; la compensación por parte de los segmentos normales cuando las partes 
de la cadena están limitadas por lesión o enfermedad, a menudo provee el continuado uso funcional de la extremidad. Debido a esta habilidad adaptativa el ferulaje en la extremidad superior debe ser cuidadosamente diseñado para prevenir inmovilidad de articulaciones que no lo requieran.

\section{Permitir la óptima sensación}

Sin sensación, la mano es perceptivamente ciega y funcionalmente limitada debido a que el estímulo cutáneo provee retroalimentación para la realización de la actividad; lo anterior, lleva a considerar que, el diseño de la férula, en lo posible, debe dejar la mayor cantidad de área palmar táctil libre de material oclusivo.

\section{Permitir una eficiente construcción y ajuste}

Diseños apropiados pueden hacer eficiente el proceso de construcción y de adaptación y con esto, controlar el tiempo y costo reduciéndolos de una manera considerable.

\section{Proporcionar una fácil aplicación y remoción}

Deben contar con un sistema sencillo para colocar y retirar. La dependencia de asistencia por parte de otros, puede recaer en frustración del usuario y la familia resultando en el establecimiento de hábitos de uso pobres y poco estructurados lo que usualmente deriva en que el usuario termine desechando la férula.

\section{Principios Específicos de Diseño}

Son aquellos que se centran en el diseño propio de la férula y se basan, en los requerimientos individuales del usuario teniendo en cuentas las consideraciones técnicas y médicas, de tal forma que apliquen al diagnostico y las necesidades terapéuticas en el proceso de intervención del usuario.

\section{Identificar las articulaciones involucradas}

Una cuidadosa evaluación de la mano, donde se tenga en cuenta la existente y potencial movilidad de las articulaciones, es quizás, la más importante consideración para determinar la última configuración de una férula de mano. Datos específicos de mediciones de segmentos corporales en relación al patrón de movimiento activo y/o pasivo que se busca, proveen al terapeuta de la información esencial que permitirán un diseño de la férula, que puede efectivamente mejorar las áreas problema. 
Después de esto, se debe determinar cuáles articulaciones van a ser inmovilizadas y cuáles recibirán esfuerzos de movilización.

\section{Revisar el propósito}

Es fundamental mantener un propósito durante todo el proceso de ferulización, ya sea que se esté pensando en inmovilizar, en incrementar el movimiento pasivo o en sustituir el movimiento activo. Después de identificar las articulaciones claves, se debe revisar el objetivo de funcionalidad específica de la férula dada.

\section{Considerar los efectos cinéticos}

Debido a que las extremidades superiores funcionan como una cadena cinética, la inclusión de una articulación en una férula puede alterar fuerzas internas y externas, articulaciones proximales y dístales. Es fundamental reconocer los problemas potenciales y entender cómo controlar y usar las fuerzas alteradas. Fuerzas incrementadas en articulaciones no inmovilizadas se convierten en un factor significativo en el diseño de férulas, especialmente, cuando las articulaciones adyacentes no pueden tolerar el estrés adicional. La anterior premisa, determina la creación de férulas que producen mínimos efectos compensatorios a estructuras proximales y dístales. Los conceptos cinéticos pueden ser también usados ventajosamente para incrementar el movimiento.

\section{Identificar áreas de disminución de la sensibilidad}

Es importante identificar áreas de disminución o ausencia de sensibilidad antes de finalizar el diseño de una férula. Debido a la alta posibilidad para generar necrosis por presión, se deben crear férulas que prevean o minimicen la acción de las fuerzas sobre aquellas áreas donde la sensibilidad está deteriorada.

\section{Determinar la superficie de aplicación de la férula}

La decisión acerca de en qué superficie o superficies de la mano o antebrazo será aplicada la férula, es el próximo paso en la progresión a través de la jerarquía de principios del plan de diseño. Esta decisión está influenciada por las relaciones mutuas de factores anatómicos y mecánicos. 


\section{Principios De Construcción}

Los principios de construcción acompañan los conceptos relacionados con la duración, la estética y el confort de la férula. La adhesión cuidadosa a estos principios proporciona un producto bien terminado, útil y que garantice su uso regular dentro de las rutinas propias del usuario.

Como los materiales para elaboración de férulas varían considerablemente en sus respectivas propiedades físicas, tanto la temperatura como los equipos empleados para fabricarlas deben ser análogos a los materiales utilizados. Para protección del usuario y del terapeuta, deben observarse precauciones de seguridad durante todas las etapas de construcción. Para lograr un buen efecto estético los bordes de la férula deben ser cuidadosamente emparejados y los extremos internos y externos de todos los componentes, los componentes de unión deben asegurarse y los ribetes o los dispositivos de seguridad deben ser terminados, eliminando puntos o superficies que puedan inadvertidamente causar lesión o enganchar la ropa. Puede proporcionarse ventilación cuando sea necesario y el acolchado interno debe asegurarse sin superficies de superposición o arrugas (HOPKINS, SMITH, 2001).

\section{Descripción de materiales utilizados en la elaboración de la férula}

\section{Metodo}

Esta propuesta se enmarca dentro de lo denominado aporte tecnológico porque busca mostrar la elaboración de una férula dinámica adaptada de tipo Tenodesis a partir de un material termoplástico de alta resistencia, de fácil manipulación, bajo costo y de fácil acceso.

Valga aclarar que, debido al hecho que el presente documento es una propuesta metodológica centrada en elaborar una Férula dinámica adaptada de tipo Tenodesis no se establece universo ni muestra.

Etapa Inicial: Construcción Teórica

Segunda Etapa: Diseño, Elaboración y Adaptación de la Férula.

Etapa Final: Presentación de Resultados. 


\section{Policloruro De Vinilo}

El Policloruro de Vinilo o PVC (del inglés Polyvinyl Chloride) es un polímero termoplástico. Se presenta como un material multicolor que comienza a reblandecer alrededor de los $80^{\circ} \mathrm{C}$ y se descompone sobre $140^{\circ} \mathrm{C}$. Cabe mencionar que es un polímero que crea repulsiones electrostáticas que reducen la flexibilidad de las cadenas poliméricas; ésta dificultad en la conformación estructural, en la mayoría de las aplicaciones, hace necesaria la incorporación de aditivos para ser obtenido un producto final deseado. El policloruro de vinilo es un material dúctil y tenaz; presenta estabilidad dimensional y resistencia ambiental. Además, es reciclable por varios métodos.

\section{Fieltro Ortopédico (Ortopedic Felt):}

Es un tejido acolchado, que proporciona suavidad y confort a los segmentos corporales que se encuentran en contacto con la ferula. Su función es evitar en el usuario puntos de presión que deriven en lesiones cutáneas tales como las úlceras por contacto y/o escaras, entre otras.

\section{Velcro o Cinta Autoadhesiva}

Conforma el sistema de correaje y consiste en un conjunto de cintas texturadas de apertura y cierre rápido. Cuenta en un lado con unos ganchos más o menos deformables (superficie macho) que se adosan a una tira de fibras enmarañadas. (Superficie hembra). En este caso se utilizó velcro de $2.5 \mathrm{~cm}$. y $5 \mathrm{~cm}$. de grosor.

\section{Solución de caucho:}

Para pegar el material de acolchado, se utilizó este tipo de pegante. La Solución de caucho, es una sustancia volátil, derivada del petróleo. Se utiliza para unir dos superficies de materiales tales como cuero, espumas, madera, entre otros.

\section{Otros:}

Para la sujeción de las piezas que aportan el dinamismo a la férula, se utilizaron remaches de aluminio tipo tubulares. Se eligieron este tipo de remaches porque permiten una suave y ajustada movilidad de la articulación, pero se garantiza un buen soporte entre las dos piezas. 


\section{SEGUNDA ETAPA:}

\section{PROTOCOLO DE ELABORACIÓN DE LA FERULA TIPO TENODESIS}

\section{Definición de la Férula Tipo Tenodesis}

Es una férula larga, dinámica que facilita la pinza trípode a partir del efecto Tenodesis natural de la muñeca, Se activa a través de movimientos de flexo-extensión de dicha articulación.

\section{Criterios de prescripción}

- $\quad$ Secuelas de cuadriplejia o lesiones a nivel de C5-C6, y que no logran realizar un movimiento voluntario de los dedos pero conservan la capacidad de flexionar y extender su muñeca.

- $\quad$ No presentar lesión en piel en el antebrazo, la mano y los dedos I a III.

- $\quad$ No presentar edema en el antebrazo, la mano y los dedos I a III.

\section{Objetivo De La Férula}

- $\quad$ Promover un agarre de pinza trípode funcional por efecto de tenodesis en personas con secuelas de lesión raquimedular a nivel de C5-C6.

- Proteger la alineación de su articulación de la muñeca.

\section{Elaboración}

Para la elaboración de la Férula Tenodesis se deben realiza los siguientes pasos:

\section{Paso 1: Toma De Medidas Antropométricas}

Se toma la medida de los contornos y longitudes de los siguientes segmentos corporales: 


\begin{tabular}{|l|l|}
\hline \multicolumn{1}{|c|}{ MEDIDA } & \multicolumn{1}{|c|}{ SEGMENTO CORPORAL } \\
\hline Medida 1 & $\begin{array}{l}\text { Contorno tercio medio del antebrazo } \\
\text { Medida 2 } \\
\text { estiloides del radio y la ulna. }\end{array}$ \\
\hline Medida 3 & $\begin{array}{l}\text { Contorno de la mano articulaciones metacarpofalangicas II } \\
\text { a V. }\end{array}$ \\
\hline Medida 4 & $\begin{array}{l}\text { Contorno de la articulación interfalangica del pulgar. } \\
\text { Medida 5 }\end{array}$ \\
\hline Medida 6 & $\begin{array}{l}\text { Longitud entre el tercio medio del antebrazo y el proceso } \\
\text { estiloides del radio. }\end{array}$ \\
\hline Medida 7 & \begin{tabular}{l} 
Longitud del tercio medio de la falange distal del pulgar. \\
\hline
\end{tabular} \\
\hline
\end{tabular}

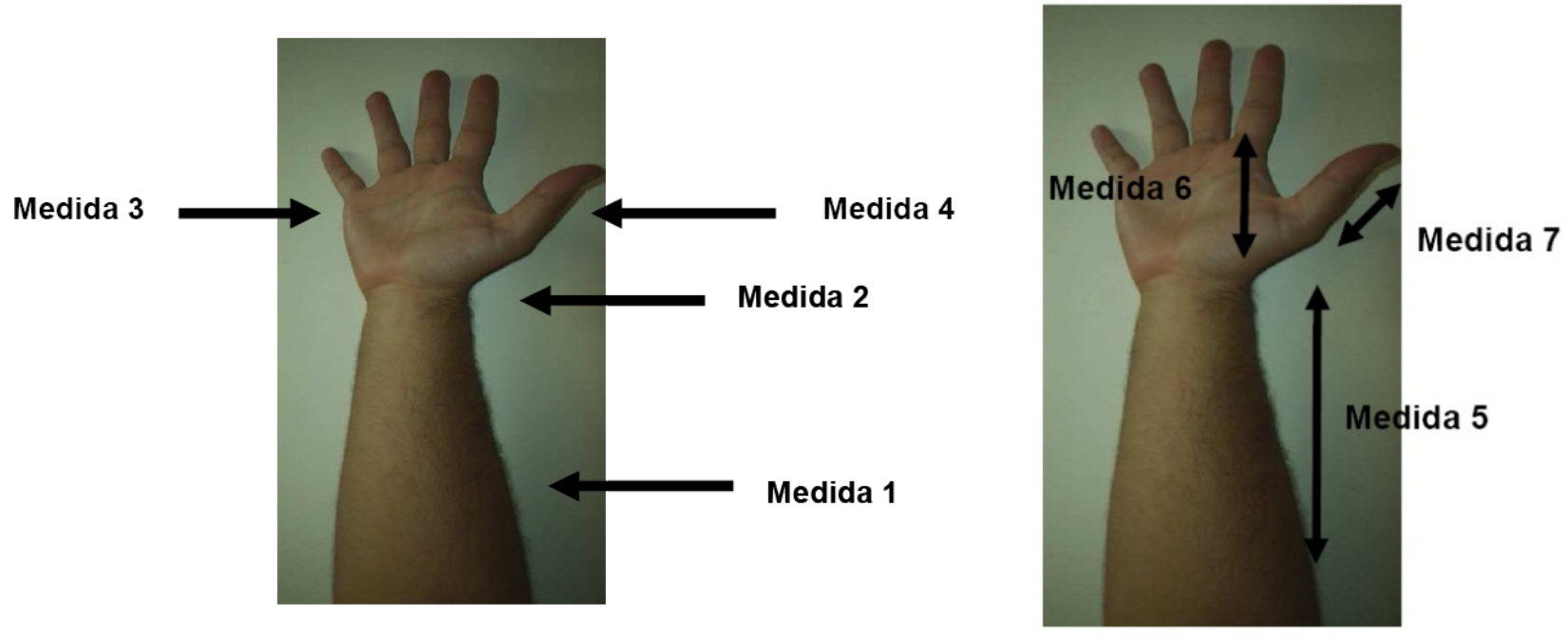


Figura 2. Mediciones antropométricas

\section{Paso 2: Cálculos Matemáticos}

Teniendo las medidas de los segmentos involucrados, se calculan las dimensiones del dispositivo, aplicando la siguiente formula al resultado de las medidas de los contornos tomados al miembro superior (medidas 1,2 y 3 ) a excepción de la pieza del pulgar la cual si conserva el valor completo del contorno del pulgar:

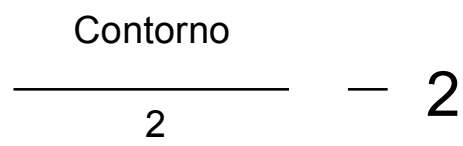

$=$ distancia del segmento

\section{Paso 3: Trazado de las Piezas}

Posteriormente se trazan los moldes de las piezas ya modificadas para la nueva férula. Este primer trazo se hace en papel teniendo en cuenta las medidas de los segmentos tomados anteriormente y los cálculos matemáticos efectuados.

Las piezas que conforman el dispositivo son:
A. Pieza de antebrazo.
B. Pieza de mano.
C. Pieza de pulgar.
D. Barra de unión.
E. Pieza de unión.

\section{Paso 4: preparación de materiales}

Se toma el tubo de termoplástico Policloruro de vinilo PVC. Se corta por la mitad longitudinalmente, para obtener dos partes de igual tamaño. Seguido a esto, se procede a 
calentar $^{2}$ una de las piezas y aplanarla, abriéndola para que quede una pequeña lámina donde sea fácil trazar los moldes de las diferentes piezas de la férula.

\section{Paso 5: Trazado de las piezas en el termoplástico}

Se pasan los moldes hechos en papel al trozo de Policloruro de vinilo PVC, utilizando, preferiblemente, un lápiz número 2 de mina gruesa.

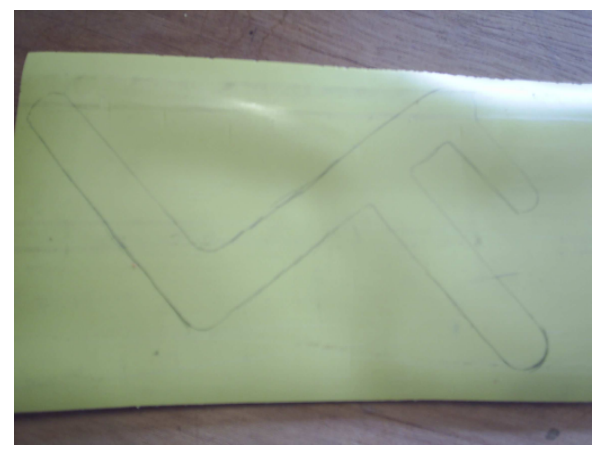

Figura 3. Pieza trazada en termoplástico

\section{Paso 6: Corte de las piezas}

Una vez las plantillas han sido dibujadas en la lámina de Policloruro de vinilo PVC, se procede a aplicar calor sobre las figuras trazadas en la lámina utilizando la pistola industrial de calor a una temperatura de $279^{\circ} \mathrm{C}$ aproximadamente durante 4 minutos, cuando el plástico está maleable, con unas tijeras de lámina se procede a cortar los contornos de las figuras. Para esta tarea, es importante tener algunas precauciones:

1. Conservar una distancia de $20 \mathrm{~cm}$ entre la pistola de calor y la superficie de la lámina porque corre el riesgo de que se queme la lámina y en el peor de los casos que se dañe la pistola por sobrecalentamiento.

2. Siempre que esté manipulando materiales con pistola de calor, es imprescindible utilizar guantes gruesos. En este caso, se utilizaron guantes de asbesto, con el objetivo de proteger las manos del intenso calor.

${ }^{2}$ El proceso de calentado de estos plásticos es a altas temperaturas, de manera que para abrirlo debe ser en un horno (230 a $240^{\circ}$ centígrados). Si la pieza de plástico es pequeña, puede abrirse calentándola con una pistola industrial de calor. 
Este procedimiento se repite 5 veces para tener así las 5 piezas que componen la férula, listas para su montaje.

Las piezas ya cortadas se pulen con una lima, cuidando de eliminar de bordes filosos y limpiar de rebaba que pueda molestar al usuario. Ya con un primer pulimento, se redondean los extremos de cada una de las piezas y se da un último pulimento general, con un Moto-tool o minitaladro ${ }^{3}$.

\section{Paso 7: Moldeado de las piezas}

Una vez pulidas las piezas se procede a moldearlas calentando cada una de ellas hasta que el material esté maleable. En este momento se procede a su manipulación para darles la forma necesaria. No sobra recordar el uso de guantes de asbesto. Hay que tener en cuenta que durante el proceso de moldeado, se deben respetar la silueta y formas anatómicas de los segmentos correspondientes esto se logra recurriendo a superficies planas y objetos cilíndricos que emulen las áreas de contacto de cada pieza.

Figura 4. Moldeado de las piezas

\section{Paso 8: ensamble de las piezas}

Una vez con los bordes redondeados y cada pieza moldeada al segmento corporal correspondiente, se procede a ensamblar las piezas utilizando remaches metálicos, hasta tenerla completamente armada.

\section{Paso 9: Limpieza de la férula}

Acto seguido, se proceda a limpiar la férula utilizando un paño y disolvente para pintura (Thinner), especialmente, para eliminar las marcas del lápiz y las marcas de fábrica propias del termoplástico Policloruro de Vinilo PVC, de ser necesario se pueden limar los bordes de nuevo utilizando el motortool.

\footnotetext{
${ }^{3}$ Un moto-tool o minitaladro es una máquina eléctrica rotativa que se puede utilizar con una sola mano. En uno de sus extremos se coloca la herramienta adecuada a cada trabajo y debido al movimiento de rotación que ésta adquiere permite realizar múltiples tareas sobre la pieza a trabajar.
} 


\section{Paso 10: Acolchado de la férula}

El paso siguiente es el acolchado de la férula. En este trabajo, se utilizó un material tipo fieltro denominado Orthopedic Felt. Para esto, se colocan los moldes trazados en papel, y se procede a pasarlos al fieltro dejando un margen de aproximadamente $0.5 \mathrm{~cm}$. de distancia de cada borde con el objetivo que el material blando sobresalga para evitar puntos de presión y bordes que pueden resultar hirientes especialmente si no hay un buen pulido. A continuación, se adhieren a la cara interna de la férula principalmente en aquellas partes que estarán en contacto con la piel utilizando pegante para caucho y o pegante instantáneo.

\section{Paso 11: colocación de correas}

Finalmente, se colocan las correas en la férula utilizando cinta velcro, la cual se ubicará en las barras estabilizadoras de la pieza del antebrazo, de la pieza de la mano y de la palanca estabilizadora de los dedos II y III.

De esta forma se da por finalizada la confección de la férula de Tenodesis.

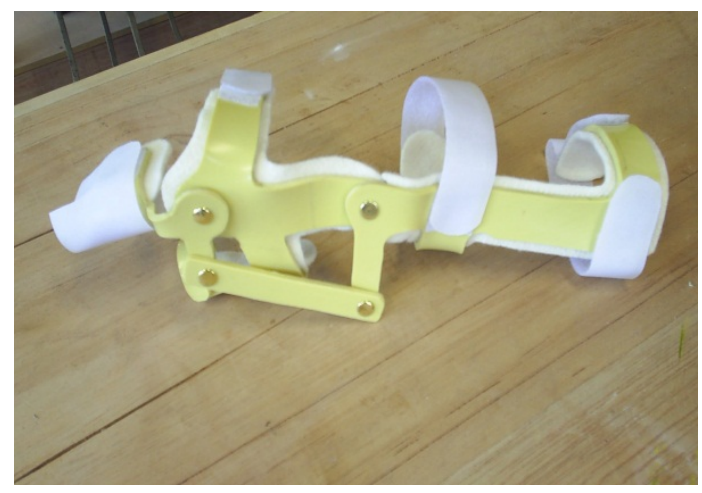

Figura 5. Colocación de correas a la férula de tipo Tenodesis. 


\section{ASPECTOS A TENER EN CUENTA}

En el marco de la intervención de Terapia Ocupacional centrada en el cliente, donde se busca satisfacer las necesidades del usuario, permitiéndole a este participar en la toma de decisiones, y, considerando que se utilizará en su realización un material termoplástico de alta resistencia, se proponen las siguientes modificaciones a algunas piezas de la férula. Dichas modificaciones, se hacen partiendo de la férula Tenodesis clásica metálica:

- Partiendo del nivel de amplitud articular que se desea proporcionar, el diseño permite el alargamiento o reducción de la barra de unión.

- Al moldear la pieza de unión, en lo relativo al segmento para posicionar dedos II y III, se puede graduar el ángulo de flexión para las articulaciones interfalángicas proximal y distal y así regular el cierre del patrón de pinza ya sea proporcionando un agarre cilíndrico con los tres primeros dedos o un agarre tipo pinza trípode.

- El segmento para posicionar dedos II y III puede ser alargado o acortado, según el número de dedos que desea movilizar, ya sea para promover movimiento de los dedos II a $\mathrm{V}$ en un agarre cilíndrico, o para movilizar solo el dedo II en pro de facilitar un agarre de pinza fina.

\section{DISCUSIÓN}

La férula tipo Tenodesis, puede ser un apoyo tecnológico importante en el proceso de adquisición de independencia y autonomía de las personas que presentan lesión raquimedular a nivel de $\mathrm{C} 5-\mathrm{C} 6$. Pese a esto, es muy poco conocida en nuestro medio, y a nivel comercial en la ciudad, no existe una oferta de este dispositivo lo que la convierte en un aparato difícil de adquirir, costoso y complicado.

La férula dinámica adaptada de tipo Tenodesis, propuesta en este trabajo, cumple con los principios biomecánicos y con los principios básicos de diseño, elaboración y adaptación, por lo cual se respalda el fundamento de diseño centrado en el usuario a partir de las mediciones antropométricas.

Los principios biofísicos en que ha sido realizada la férula permite la oposición adecuada de los dedos II y III con el dedo pulgar, con lo cual se facilita un agarre de pinza trípode funcional.

Las características y descripción del diseño de esta férula dinámica adaptada tipo Tenodesis permiten el fácil ajuste frente a las características de la mano del usuario que la requiera ya que ha sido confeccionada respetando los valores antropométricos, sumado al 
hecho que dicho ajuste es proporcionado por el material de acolchado utilizado el cual se adapta a las curvaturas corporales.

La férula dinámica adaptada tipo Tenodesis, es un dispositivo de fácil adquisición para quien han padecido de trauma raquimedular $\mathrm{C} 5-\mathrm{C} 6$, debido a que los materiales en que está construida, son de fácil adquisición y a muy bajo costo lo que respecta al costo del material utilizado, se puede decir que no se invierte más de $\$ 10.000$ COP (\$5.5 USD aprox.), lo cual le proporciona una marcada ventaja comparado con materiales como Solaris ${ }^{\mathrm{TM}} 1 / 8$, Prism $^{\mathrm{TM}}$ $1 / 8$ ", Encore ${ }^{\mathrm{TM}} 1 / 8$ " entre otros cuyo valor a la fecha varía desde $\$ 93.000$ COP hasta $\$$ 120.000 COP ( $\$ 52$ USD y $\$ 67$ USD) por hoja de $46 \mathrm{~cm} \times 61 \mathrm{~cm}$.

Otro aspecto que se debe incluir dentro del precio de la férula, mas no por esto debe convertirse en un factor que aumente excesivamente los costos, es la experticia y conocimientos del terapeuta para ofrecer un dispositivo pertinente y de excelente calidad. En este caso, \$14.500 COP por sesión (Decreto 2423 del 31 de Diciembre de 1996, Actualizado a 2012) aproximadamente 4 sesiones para elaboración de la férula, es decir $\$ 58.000$ COP (\$34.8 USD aprox.); lo que conlleva a un valor total del dispositivo de $\$ 68.000$ COP (\$40.8 USD aprox.).

El diseño de la férula dinámica adaptada de tipo Tenodesis, permite la alineación funcional de la muñeca evitando desviaciones de tipo radial o ulnar. Además, por su diseño a partir de barras transversales y longitudinales, y por ir completamente acolchonada, presenta un bajo riesgo de producir lesiones cutáneas y puntos de presión, así como permite una excelente ventilación.

\section{AGRADECIMIENTOS}

De una manera corta, pero no por eso carente de valor, la elaboración de este proyecto se debe a las Terapeutas Ocupacionales Patricia Murillo y Liliana Tenorio quienes guiaron mi proceso formativo y me enseñaron lo importante de la perseverancia. 


\section{REFERENCIAS BIBLIOGRAFICAS}

ACOSTA, D. C. (2007) Manual multimedia de diseño, elaboración y adaptación de tres férulas básicas para la mano. Proyecto para optar al título de terapeuta ocupacional. Escuela de rehabilitación humana. Programa de Terapia Ocupacional. Cali, Colombia.

HOPKINS, H. L., SMITH H. D. (2001/2005) Terapia Ocupacional. 10 edición. Madrid España. Editorial médica Panamericana, 29, 305.

LASERNA, R. (2002). Tecnología Y Autonomía Personal: Aproximaciones Prácticas. Revista de la ocupación humana on-line. Volumen 9, numero 3. 16-17.

MALKUSH, M. Hand and Wrist-Hand Orthoses. Disponible en:

http://atwiki.assistivetech.net/index.php/Hand_and_Wrist-Hand_Orthoses.

TROMBLY, A. C. (1990/2001) Terapia ocupacional para enfermos incapacitados físicamente. Baltimore, Lippincott. ED. Científicas.

TURNER, A. (2002) Terapia Ocupacional y disfunción física. España. Editorial Elsevier science, 424, 426. 